POS $\quad \begin{aligned} & \text { PROCEEDINGS } \\ & \text { OF SCIENCE }\end{aligned}$

\title{
Higgs boson and Top quark masses as tests of Electroweak Vacuum Stability
}

\author{
Isabella MASINA* \\ (University of Ferrara and INFN Sez. di Ferrara) \\ E-mail: masina@fe.infn.it
}

\begin{abstract}
The measurements of the Higgs boson and top quark masses can be used to extrapolate the Standard Model Higgs potential at energies up to the Planck scale. Adopting a Next-to-Nextto-Leading Order renormalization procedure, we argue that electroweak vacuum stability is at present allowed, discuss the associated theoretical and experimental errors and the prospects for its future tests.
\end{abstract}

XXI International Workshop on Deep-Inelastic Scattering and Related Subject -DIS2013, 22-26 April 2013

Marseilles, France

\footnotetext{
* Speaker.
} 


\section{Introduction}

July 4th 2012 was an historical date in particle physics as of the CERN ATLAS [1] and CMS [2] collaborations announced the discovery of a particle like the Standard Model (SM) Higgs boson. The couplings of this new particle are yet to be precisely tested but, at present, they indeed have been find in agreement with those expected for a SM Higgs boson. The mass of the new particle is already quite accurately determined: adding in quadrature statistical and systematic errors, it turns out to be in the range $124.8-126.5 \mathrm{GeV}$ at $2 \sigma$.

Here we assume that the new particle is actually the SM Higgs boson and, following ref. [3], discuss the implications that its mass value, together with other relevant parameters such as the top quark mass and the strong gauge coupling, have on the behavior of the Higgs potential at very high energy scales, in particular for the sake of electroweak vacuum stability.

The project of extrapolating the Higgs potential up to the Planck scale is a long standing one $[4,5,6]$, and was revamped in the fall of 2011 [7, 8, 9, 10] after the first LHC hints of a Higgs boson. Recently, the tools for a Next-to-Next-to-Leading Order renormalization procedure were derived $[11,12,13,14]$. So, there are now all the ingredients to carry on this long standing project. Clearly, the extrapolation is based on the assumption that there is a desert up to the Planck scale or, better, that possible new physics do not affect significantly the running of the Higgs quartic coupling, dominating the Higgs potential at high energy.

It is interesting that the recently discovered experimental Higgs mass range, combined with the experimental top mass range, indicates a particularly intriguing high energy behavior of the Higgs potential, close to the transition between electroweak vacuum stability and metastability. This is due to the fact that, for these Higgs and top mass values, the Higgs quartic coupling can be very small or even negative. Since the dependence on the top mass is strong and quite subtle, it is not surprising that different groups slightly disagree in the interpretation of the results, some of them favoring [13] and some other disfavoring [14] electroweak vacuum stability.

Traditionally the top pole mass was used in the analysis, but it has been pointed out [15] that the top pole mass value used in previous analyses and taken to be the one measured at Tevatron, $m_{t}^{\text {exp }}=173.2 \pm 0.9 \mathrm{GeV}$ [16], is not unambiguously derived and that a more careful derivation should be rather based on the running top mass in the $\overline{\mathrm{MS}}$ scheme, $\overline{m_{t}}\left(m_{t}\right)=163.3 \pm 2.7 \mathrm{GeV}$. As shown in [15], the top pole mass range consistently derived from the running one, $m_{t}=173.3 \pm 2.8$ $\mathrm{GeV}$, is plagued by a larger error than the Tevatron measurement considered in [14], rescuing electroweak vacuum stability. In our analysis [3] we keep as a free parameter the running top mass, rather than the pole one. In this way we completely avoid the theoretical uncertainties associated to the top Yukawa matching procedure.

\section{Stability or metastability}

The experimental region of the values of the Higgs and top masses is very intriguing form the theoretical point of view, since the Higgs quartic coupling $\lambda(\mu)$ could be rather small, vanish or even turn negative at a scale $\mu$ slightly smaller than the Planck scale. Accordingly, the behavior of the Higgs potential at high energy changes drastically: if $\lambda(\mu)$ is always positive, the electroweak vacuum is a global minimum, possibly accompanied by another local minimum just below the 
Planck scale, which could have played a role in primordial inflation [8, 17, 18]; if $\lambda(\mu)$ turns negative below $M_{\mathrm{Pl}}$, the electroweak vacuum correspondingly becomes metastable [5, 6].

These drastically different possibilities for the behavior of the renormalization improved Higgs potential at high energy are illustrated in fig. 1 , for $m_{H}=126 \mathrm{GeV}$ and selected values of the running top mass $\overline{m_{t}}\left(m_{t}\right)$. Let start considering $\overline{m_{t}}\left(m_{t}\right)=161.989 \mathrm{GeV}$. Increasing the latter by just 1 $\mathrm{MeV}$, the potential develops an inflection point; the associated $\lambda(\mu)$ becomes as small as $\mathscr{O}\left(10^{-5}\right)$. Increasing again $\overline{m_{t}}\left(m_{t}\right)$ by about $200 \mathrm{keV}$, the minimum of $\lambda(\mu)$ is equal to zero: a second vacuum degenerate with the electroweak one is obtained. Further increasing $\overline{m_{t}}\left(m_{t}\right)$ makes $\lambda(\mu)$ turn negative: the electroweak vacuum becomes metastable.

We now turn to the determination of the line in the plane $\left[m_{H}, \overline{m_{t}}\left(m_{t}\right)\right]$ separating the stability from the metastability region. In the lower part of fig. $2 \lambda(\mu)$ is always positive, while in the upper part it becomes negative before reaching the Planck scale.

The transition line of fig. 2 is obtained with the input parameter values discussed in [3] and by matching the running Higgs quartic coupling at $m_{H}$. Clearly it is crucial to estimate the theoretical error associated to experimental ranges of the input parameters and the one associated to the matching procedure; here we summarize the results presented in [3]. Since stability can be achieved in the whole experimental range for $m_{H}$ (shaded vertical region), but this is not the case for $\overline{m_{t}}\left(m_{t}\right)$ (shaded horizontal region), it is convenient to write down the condition of electroweak vacuum stability under the form of an upper bound on the top mass:

$$
\overline{m_{t}}\left(m_{t}\right) \leq 162.0+0.47\left(m_{H}-126\right) \pm 0.7_{\alpha_{3}}-0.2_{\mu_{\lambda}}
$$

where masses are in $\mathrm{GeV}$. The first error (oblique arrow in fig. 2) is associated to the experimental error on the strong gauge coupling $\alpha_{3}\left(m_{Z}\right)$ and turns out the be larger than the second error (horizontal arrow in fig. 2), that accounts for the theoretical error associated to the Higgs quartic coupling matching $[13,14]$.

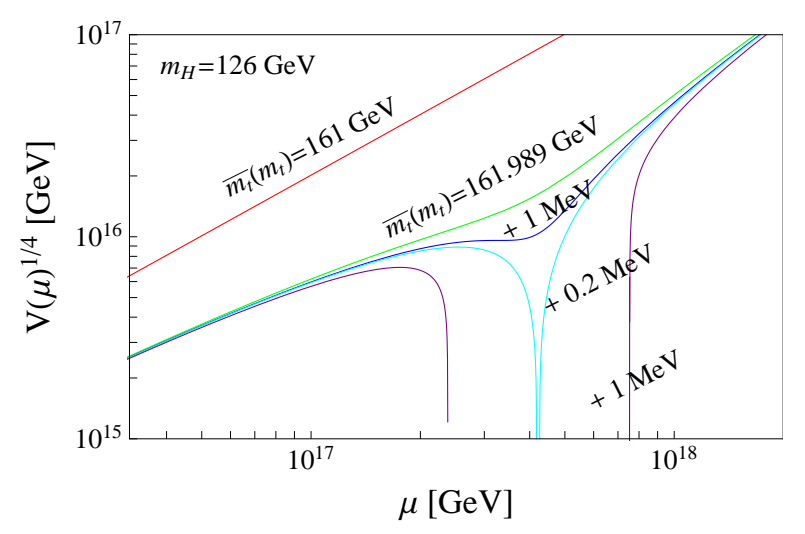

Figure 1: The SM Higgs potential as a function of the renormalization scale $\mu$, for $m_{H}=126 \mathrm{GeV}$ and increasing values of $\overline{m_{t}}\left(m_{t}\right)$. The other input parameters are fixed at the central values discussed in [3]. 


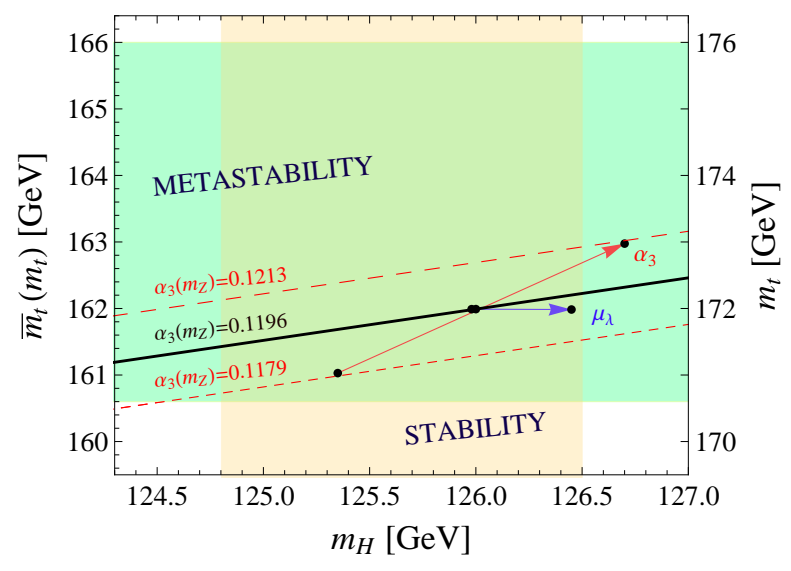

Figure 2: The solid (black) line marks the points in the plane $\left[m_{H}, \overline{m_{t}}\left(m_{t}\right)\right]$ where a second vacuum, degenerate with the electroweak one, is obtained just below the Planck scale. The (red) diagonal arrow shows the effect of varying $\alpha_{3}\left(m_{Z}\right)=0.1196 \pm 0.0017$; the (blue) horizontal one shows the effect of varying $\mu_{\lambda}$ (the matching scale of $\lambda$ ) from $m_{Z}$ up to $2 m_{H}$. The shaded (yellow) vertical region is the $2 \sigma$ ATLAS [1] and CMS [2] combined range; the shaded (green) horizontal region is the range $\overline{m_{t}}\left(m_{t}\right)=163.3 \pm 2.7 \mathrm{GeV}$, equivalent to $m_{t}=173.3 \pm 2.8 \mathrm{GeV}[15]$.

\section{Discussion and prospects}

Given the present experimental range of the running top mass [15], fig. 2 shows that stability of the SM electroweak vacuum is allowed for the whole experimental Higgs mass range [3].

In order to understand whether we live in a stable or metastable vacuum it is then crucial to better determine $\overline{m_{t}}\left(m_{t}\right)$. As discussed in [15], after LHC the Higgs mass will presumably be known with an accuracy of $\mathscr{O}(100) \mathrm{MeV}$ [19], but the precision on the top mass would improve only by a factor of two. For instance, if the whole range of $\overline{m_{t}}\left(m_{t}\right)$ below $163 \mathrm{GeV}$ would be excluded, we would conclude that our vacuum is metastable; otherwise the investigations should continue.

A self-consistent and precise determination of the top quark mass can best be performed at a high-energy electron-positron collider, with a planned accuracy of $\mathscr{O}(100) \mathrm{MeV}$. Moreover, at an electron-positron collider $\alpha_{3}\left(m_{Z}\right)$ could be determined with an accuracy close to or better than $\Delta \alpha_{3}\left(m_{Z}\right)=0.0007$ (this precision is sometimes currently adopted [13, 14] but cannot be considered to be conservative according to ref. [15]). At this stage, if the stability region will still have an overlap with the allowed ranges of the top and Higgs masses, we will be mostly limited by the theoretical uncertainty associated to the matching scale of the Higgs quartic coupling.

Notice that the possibility to be in a stable Higgs potential configuration is particularly relevant for cosmology. For instance, a shallow false minimum close to the Planck scale where the Higgs field could have been trapped for some period, could have been responsible for primordial inflation [8, 17, 18]; such configuration is realized only if the Higgs quartic coupling and its derivative satisfy the very specific boundary conditions derived in ref. [3], which possibly have a deep origin in quantum gravity. Stability is a necessary condition also in models of Higgs inflation with non-minimal gravitational couplings, see for instance [13] and references therein. 
As a final comment, we stress that new physics in addition to the SM is required to explain neutrino masses and mixings (and also dark matter). The mechanism responsible for neutrino masses could affect the Higgs quartic coupling. The impact that the inclusion of neutrino masses via a type I seesaw has on electroweak vacuum stability, in particular for the sake of a shallow false minimum configuration, is discussed in [3]: a conservative upper bound on type I seesaw right-handed neutrino masses can be derived, which strongly depends on the top mass.

\section{References}

[1] G. Aad et al. [ATLAS Collaboration], Phys. Lett. B 716 (2012) 1

[2] S. Chatrchyan et al. [CMS Collaboration], Phys. Lett. B 716 (2012) 30

[3] I. Masina, Phys. Rev. D 87 (2013) 053001

[4] P. Q. Hung, Phys. Rev. Lett. 42 (1979) 873. N. Cabibbo, L. Maiani, G. Parisi and R. Petronzio, Nucl. Phys. B 158 (1979) 295. M. Lindner, Z. Phys. C 31 (1986) 295. M. Lindner, M. Sher and H. W. Zaglauer, Phys. Lett. B 228 (1989) 139. M. Sher, Phys. Rept. 179 (1989) 273. M. Sher, Phys. Lett. B 317, 159 (1993) [Addendum-ibid. B 331, 448 (1994)]. G. Altarelli and G. Isidori, Phys. Lett. B 337, 141 (1994). C. D. Froggatt and H. B. Nielsen, Phys. Lett. B 368 (1996) 96. B. Schrempp and M. Wimmer, Prog. Part. Nucl. Phys. 37 (1996) 1.

[5] J. A. Casas, J. R. Espinosa and M. Quiros, Phys. Lett. B 342, 171 (1995). J. R. Espinosa and M. Quiros, Phys. Lett. B 353, 257 (1995). J. A. Casas, J. R. Espinosa and M. Quiros, Phys. Lett. B 382 (1996) 374.

[6] G. Isidori, G. Ridolfi and A. Strumia, Nucl. Phys. B 609 (2001) 387. J. R. Espinosa, G. F. Giudice and A. Riotto, JCAP 0805 (2008) 002. G. Isidori, V. S. Rychkov, A. Strumia and N. Tetradis, Phys. Rev. D 77 (2008) 025034. N. Arkani-Hamed, S. Dubovsky, L. Senatore and G. Villadoro, JHEP 0803 (2008) 075. J. Ellis, J. R. Espinosa, G. F. Giudice, A. Hoecker and A. Riotto, Phys. Lett. B 679 (2009) 369.

[7] M. Holthausen, K. S. Lim and M. Lindner, JHEP 1202 (2012) 037.

[8] I. Masina and A. Notari, Phys. Rev. D 85 (2012) 123506.

[9] J. Elias-Miro, J. R. Espinosa, G. F. Giudice, G. Isidori, A. Riotto and A. Strumia, Phys. Lett. B 709 (2012) 222.

[10] Z. -z. Xing, H. Zhang and S. Zhou, Phys. Rev. D 86 (2012) 013013.

[11] L. N. Mihaila, J. Salomon and M. Steinhauser, Phys. Rev. Lett. 108 (2012) 151602.

[12] K. G. Chetyrkin and M. F. Zoller, JHEP 1206 (2012) 033.

[13] F. Bezrukov, M. Y. .Kalmykov, B. A. Kniehl and M. Shaposhnikov, JHEP 1210 (2012) 140.

[14] G. Degrassi, S. Di Vita, J. Elias-Miro, J. R. Espinosa, G. F. Giudice, G. Isidori and A. Strumia, JHEP 1208 (2012) 098.

[15] S. Alekhin, A. Djouadi and S. Moch, Phys. Lett. B 716 (2012) 214.

[16] Tevatron Electroweak Working Group and CDF and D0 Collaborations, arXiv:1107.5255 [hep-ex].

[17] I. Masina and A. Notari, Phys. Rev. Lett. 108 (2012) 191302.

[18] I. Masina and A. Notari, JCAP 1211 (2012) 031.

[19] G. Aad et al. [ATLAS Collaboration], arXiv:0901.0512 [hep-ex]. G. L. Bayatian et al. [CMS Collaboration], J. Phys. G G 34 (2007) 995. 